\title{
Efeito da Terapia Virtual na Reabilitaçáo Motora do Membro Superior de Crianças Hemiparéticas
}

\author{
Effect of Virtual Therapy on Upper Limb Motor Rehabilitation of Children With Hemiparesis \\ Anelise Vilas Bôas', Walter Luiz Magalhäes Fernandes², \\ Andréia Maria Silva ${ }^{3}$, Adriana Teresa Silva ${ }^{4}$
}

\begin{abstract}
RESUMO
Objetivo. Este estudo teve como objetivo investigar o efeito da terapia virtual em habilidades motoras do membro superior em crianças hemiparéticas. Método. A amostra foi composta de três crianças hemiparéticas $(8,66 \pm 2,30$ anos). O Inventário de Avaliação Pediátrica de Incapacidade (PEDI) e Inventário de Atividade Motora (MAL) foram utilizados para a avaliação inicial e final, também foi contado o número de acertos em cada jogo. Para o tratamento, foi utilizado um hardware (video game) marca Nintendo, modelo Wii com Wii Sports software, com os jogos (tênis, beisebol, golfe, boxe). A terapia durou 1 hora e 10 para um período de duas semanas consecutivas. Resultados. No PEDI houve diferença estatística em duas crianças $(\mathrm{p}=0,00)$; no MAL com relaçáo a qualidade de uso do membro superior houve diferença estatística em duas crianças $(p=0,00)$; com relação a frequência de uso houve diferença estatística nas três crianças $(p=0,00)$; houve um aumento do número de acertos de todos os jogos em todas as crianças. Conclusáo. Conclui-se que o uso da Realidade Virtual pode promover ganho funcional no membro superior de hemiparéticos destas crianças.
\end{abstract}

Unitermos. Reabilitação, Hemiparesia, Função.

Citação. Bôas AV, Fernandes WLM, Silva AM, Silva AT. Efeito da Terapia Virtual na Reabilitação Motora do Membro Superior de Crianças Hemiparéticas.

\begin{abstract}
Objective. This study aimed to investigate the effect of virtual therapy on the motor ability of hemiparetic children's upper limb. Method. The sample consisted of three hemiparetic children ( $8.66 \pm 2.30$ years). The Pediatric Evaluation of Disability of Inventory (PEDI) and Motor Activity Inventory (MAL) were used for initial and final assessments and the number of hits in each game was counted as well. For the treatment, a hardware (video game) was used, Nintendo brand, model Wii Sports software, using the games (tennis, baseball, golf, boxing). During a period of two weeks, the therapy lasted for 1 hour and 10 minutes each day, consecutively. Results. There was statistical difference in two children in the PEDI $(p=0,00)$ and in the MAL, related to the quality of the upper limb's use $(\mathrm{p}=0,00)$ where as relation to the frequency of use, there was statistical difference in all three children in all four games had a increase. Conclusion. It was concluded that the use of Virtual Reality can promote functional gain for hemiparetic children's upper limb.
\end{abstract}

Keywords. Rehabilitation, Hemiparesis, Function.

Citation. Bôas AV, Fernandes WLM, Silva AM, Silva AT. Effect of Virtual Therapy on Upper Limb Motor Rehabilitation of Children With Hemiparesis.
Trabalho realizado na Universidade do Vale do Sapucaí -UNIVÁS, Pouso Alegre-MG, Brasil.

1.Fisioterapeuta graduada na Universidade do Vale do Sapucaí (UNIVÁS), Pouso Alegre-MG, Brasil.

2.Médico, residência médica em Neurologia Infantil pela UNICAMP, Campinas-SP, Brasil.

3.Fisioterapeuta, docente do curso de Fisioterapia da Universidade do Vale do Sapucaí - UNIVÁS e Universidade Federal de Alfenas- UNIFAL, Alfenas-MG, Brasil.

4.Fisioterapeuta, docente do curso de Fisioterapia da Universidade do Vale do Sapucaí - UNIVÁS e Universidade Federal de Alfenas- UNIFAL, Alfenas-MG, Brasil.
Endereço para correspondência:

Adriana Teresa Silva

Av. Pref. Tuany Toledo, 470 CEP 37550-000, Pouso Alegre, MG, Brasil. E-mail: adrianat.silva@yahoo.com.br

Relato de Caso Recebido em: 16/08/11 Aceito em: 24/08/13 Conflito de interesses: nấo 


\section{INTRODUÇÃO}

O Sistema Nervoso Central (SNC) apresenta uma localização privilegiada e tem funçóes importantes, porém não está imune de apresentar doenças e lesôes traumáticas. Inúmeras doenças podem acometer o SNC da criança. Tais doenças podem ser congênitas ou adquiridas, destacando entre elas a paralisia cerebral (PC) e o trauma cranioencéfalico (TCE). O quadro clínico, a evolução e o prognóstico das lesôes são muito variáveis, sendo a hemiparesia um dos sinais clínicos mais evidentes nas lesões do $\mathrm{SNC}^{1,2}$.

A hemiparesia interfere no funcionamento motor normal, contribuindo para uma posição de assimetria postural, distribuição de peso menor sobre o lado afetado, déficits como perda da força muscular, destreza e coordenação motora ${ }^{1}$, afetando a sua independência e a qualidade de vida ${ }^{3-5}$.

A prevalência atual de $\mathrm{PC}$ na população mundial atinge 1,5 a 2,5 indivíduos a cada 1.000 nascidos vivos. No Brasil, a cada 1.000 crianças que nascem, sete são portadoras de $\mathrm{PC}^{6,7}$. $\mathrm{O}$ trauma corresponde à principal causa de morte em crianças, sendo um problema comum na infância principalmente acima dos cinco anos de idade, responsável por mais de $75 \%$ das mortes na infância ${ }^{8}$, com alto índice de internação hospitalar e significativa taxa de morbidade e mortalidade, além de sequelas e alteraçôes motoras?.

Nas décadas mais recentes tem-se observado uma crescente disseminação de tecnologias de informação em diversos setores da sociedade ${ }^{10}$. O homem vem desenvolvendo uma série de dispositivos tecnológicos (hardware e software) através dos quais, pretende ampliar a imersão e interação (visual, tátil, olfativa e gustativa) onde o utilizador interage com o ambiente virtual ${ }^{11}$. A área de saúde vem sendo impulsionada pelas novas tecnologias associadas aos programas de reabilitação. A Realidade Virtual (RV) é uma destas tecnologias que vem sendo aplicada para o treinamento de pessoas com comprometimento neurológico ${ }^{10}$.

A RV poderá ser realizada através de programas de exercícios baseados em jogos virtuais, contribuindo de maneira lúdica para a facilitação do treinamento funcional ${ }^{12}$. É de extrema importância oferecer um ambiente solicitador, que promova autonomia e diferentes possibi- lidades de descobertas e estímulos, uma vez que, o SNC de crianças apresenta com maior capacidade de gerar plasticidade, e é potencialmente mais maleável para um redirecionamento dos circuitos neuronais ${ }^{13,14}$.

O propósito do presente estudo foi verificar o efeito da Terapia Virtual nas habilidades motoras do membro superior de crianças com hemiparesia.

\section{MÉTODO}

Este estudo foi aprovado pelo Comitê de Ética em Pesquisa da Faculdade de Fisiologia, Ciências e Letras Eugênio Pacelli, da Universidade do Vale do Sapucaí com o protocolo No 278/09. Os pais das crianças foram informados sobre os objetivos e procedimentos a serem adotados e foram convidados a participarem da pesquisa e assinaram o Termo de Consentimento Livre e Esclarecido. Este pesquisa respeita as normas e diretrizes da Resolução no 196/96 do Conselho Nacional de Saúde (CNS).

\section{Amostra}

A amostra foi constituída por três crianças de gênero feminino, com idade $8,66 \pm 2,3$ anos, duas com diagnóstico clínico de Paralisia Cerebral e uma com Trauma Cranioencefálico, todas com o diagnóstico neurofuncional de hemiparesia com predomínio braquial. A coleta de dados e os atendimentos foram realizados no laboratório de motricidade humana da Faculdade de Ciências da Saúde Dr. José Antônio Garcia Coutinho/FACIMPA/ UNIVÁS.

\section{Instrumentos para avaliaçáo e atendimento}

Os instrumentos utilizados para avaliação foram: o Inventário de Avaliação Pediátrica de Incapacidade (PEDI) (no presente estudo utilizou-se somente a parte I - Habilidades Funcionais - nesta parte informa sobre o desempenho de habilidades da criança) a pontuação máxima é de 73 pontos ${ }^{15-17}$ e Inventário de Atividade Motora (MAL) para quantificar as capacidades funcionais da extremidade superior, durante realização de atividades cotidianas. É constituído de 30 questôes relacionadas à funcionalidade do membro superior afetado, possuindo dois tipos de escalas que são pontuadas pelo paciente com valores de 0 a 5: a escala de frequência de uso (150 pontos) e a escala da qualidade do movimento (150 pontos) 
onde, quanto maior a pontuação, melhor o resultado obtido $^{18-21}$. Todas as crianças foram avaliadas e reavaliadas pelo mesmo examinador.

Um vídeo-game Nintendo (modelo Wii - software Wii Sports) foi o instrumento utilizado no atendimento. Os jogos escolhido foram: tênis, baseball, boxe e golf. Todos os jogos foram transmitidos em uma tela de televisão de 29 polegadas da marca CCE.

\section{Procedimento}

O procedimento consistiu de:

1- Alongamento ativo assistido no início e final de cada sessão dos membros superiores (peitoral, tríceps, romboide e flexores e extensores de punho), com duração de 30 segundos em cada músculo; 2- O pesquisador demonstrou o manuseio correto de cada jogo (tênis, baseball, boxe e golf); 3- O paciente realizou as atividades através do comando verbal dado pelo pesquisador, onde este corrigiu, quando necessário, a postura do paciente, levando-o a execução de movimentos adequados e harmoniosos; 4- Na realização de cada atividade, durante os quatro jogos (tênis, baseball, boxe e golf) os participantes permaneceram na posição ortostática. Os pacientes que apresentavam dificuldade para segurar o controle do vídeo-game foi adaptada uma faixa elástica que envolvia a mão do mesmo. Essa faixa adaptada oferecia maior segurança e eficiência durante os jogos.

O tratamento durou duas semanas consecutivas totalizando 10 sessóes realizadas de segunda a sexta-feira exceto sábado e domingo, foram feitas 2 sessões extras para avaliação e reavaliação. Duas crianças, uma com PC e a outra com TCE realizaram a terapia no período da manhã, e a terceira com PC no período da tarde. Náo houve necessidade de intervalos/pausas entre os jogos. Totalizando uma hora e dez minutos de terapia diária. Durante a realização dos jogos foi contabilizado o número de acertos em cada sessão.

\section{Procedimentos estatístico}

A estatística descritiva foi utilizada para caracterização da amostra. Os dados foram analisados por meio do cálculo da média e desvio padrão em cada escala PEDI e MAL. Foi realizado o teste de K-S para determinar a normalidade dos dados. Em seguida utilizou o teste de
Wilcoxon das escalas de MAL e PEDI para comparação antes e após a intervenção de cada indivíduo. Todas as análises foram executadas usando o programa SPSS versão 20.0. O nível de significância adotado foi p<0,05.

\section{RESULTADOS}

Observa-se na Tabela 1 os dados clínicos das pacientes. Duas pacientes eram acometidas do hemicorpo direito e uma do hemicorpo esquerdo, todas eram do gênero feminino, duas pacientes apresentavam paralisia cerebral e uma traumatismo crânio encefálico.

Tabela 1

Valores da média, desvio padrão e percentagem das variáveis como idade, hemicorpo acometido, gênero e tipo de lesão

\begin{tabular}{ccccc}
\hline & Idade & $\begin{array}{c}\text { Hemicorpo } \\
\text { Acometido }\end{array}$ & Gênero & Tipo de lesão \\
\hline P1 & 10 & $\mathrm{D}$ & $\mathrm{F}$ & PC \\
P2 & 6 & $\mathrm{E}$ & $\mathrm{F}$ & TCE \\
P3 & 10 & $\mathrm{D}$ & $\mathrm{F}$ & PC \\
\hline & $8,6 \pm 2,3$ & $66,66 \% \mathrm{D}$ & $100 \%$ & - \\
& & $33,33 \% \mathrm{E}$ & & \\
\hline
\end{tabular}

P (1, 2 e 3) - pacientes; D - direita; E - esquerda; F: feminino; PC: Paralisia Cerebral, TCE: Traumatismo Craniano Encefálico.

$\mathrm{Na}$ Figura 1 é demonstrado os valores das médias, desvio padrão e p dos escores obtidos de cada paciente da escala de PEDI. Nota-se que houve diferença estatística na paciente dois e três $(\mathrm{p}=0,00)$. Na paciente um $(\mathrm{p}=0,15)$ não houve diferença estatística.

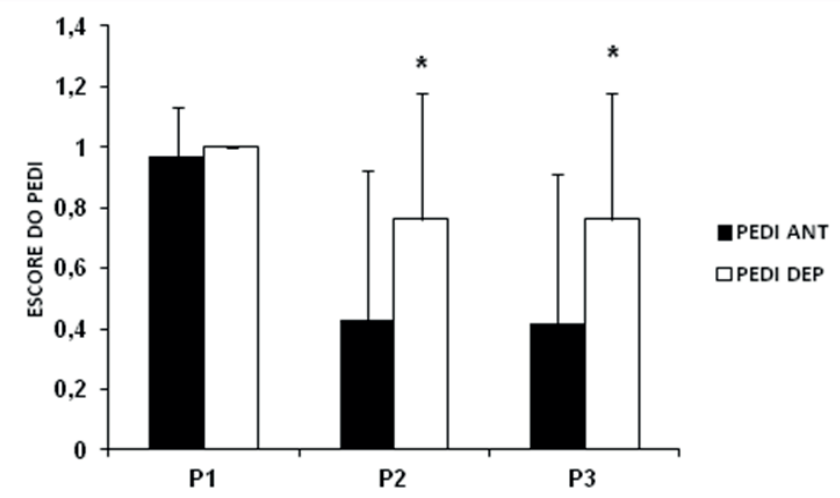

Figura 1. demostra a média e desvio padrão dos escores obtido de cada paciente pelq escala de PEDI. *Houve diferença estatística. P1: Paciente 1, P2: paciente 2; P3: paciente 3; PEDI ANT: Inventário de Avaliação Pediátrica de Incapacidade antes, PEDI DEP: Inventário de Avaliação Pediátrica de Incapacidade depois. 
Na Figura 2, observa-se os valores das médias, desvio padrão e p dos escores obtidos de cada paciente da escala de MAL com relação a qualidade de uso do membro superior. Pode-se notar diferença estatística na paciente dois e três $(\mathrm{p}=0,00)$. Na paciente um $(\mathrm{P}=0,15)$ não houve diferença estatística.

$\mathrm{Na}$ Figura 3, observa-se os valores das médias, desvio padrão e $\mathrm{p}$ dos escores obtidos de cada paciente da escala de MAL com relação a Frequência de uso do membro superior. Pode-se notar diferença estatística nas três paciente $(\mathrm{p}=0,00)$.

Na Figura 4, observa-se os valores do número de acertos dos jogos no início e final das sessôes para os jogos de tênis e baseball dos pacientes da pesquisa. Nota-se um aumento no número de acertos.

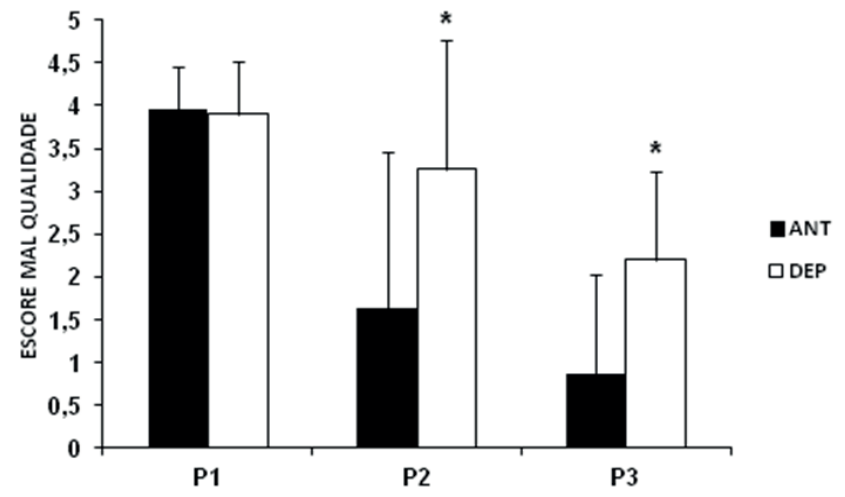

Figura 2. demostra a média e desvio padrão dos escores obtido de cada paciente da escala de MAL na qualidade do movimento. ${ }^{*}$ Houve diferença estatística. P1: Paciente 1, P2: paciente 2; P3: paciente 3; ANT: Antes; DEP: Depois.

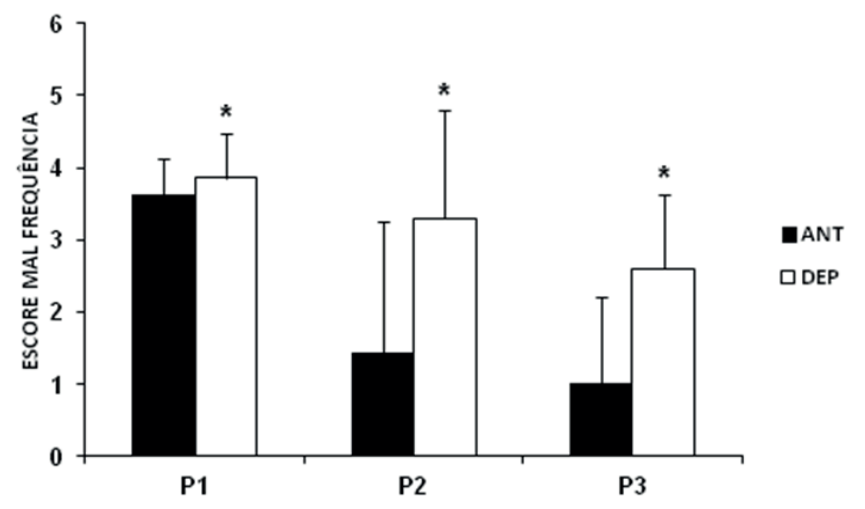

Figura 3. demostra a média e desvio padrão dos escores obtido de cada paciente da escala de MAL na frequência de uso. *Houve diferença estatística. P1: Paciente 1, P2: paciente 2; P3: paciente 3; ANT: Antes; DEP: Depois.
$\mathrm{Na}$ Figura 5, observa-se os valores do número de acertos dos jogos no início e final das sessóes para os jogos de golf e boxe dos pacientes da pesquisa. Nota-se um aumento no número de acertos.

\section{DISCUSSÃO}

A RV é uma das novas estratégias que vem sendo empregadas para reabilitação motora do membro superior. Os ambientes virtuais oferecem vantagens para reabilitação simulando situaçóes e tarefas da vida real proporcionando maior motivação. Flynn et al. ${ }^{22}$ analisaram os efeitos da realidade virtual em 12 sujeitos com lesão do SNC e concluíram que houve um aumento na produção da força dos dedos, melhora nas habilidades funcionais, aumento da agilidade com diminuição do tempo na exe-

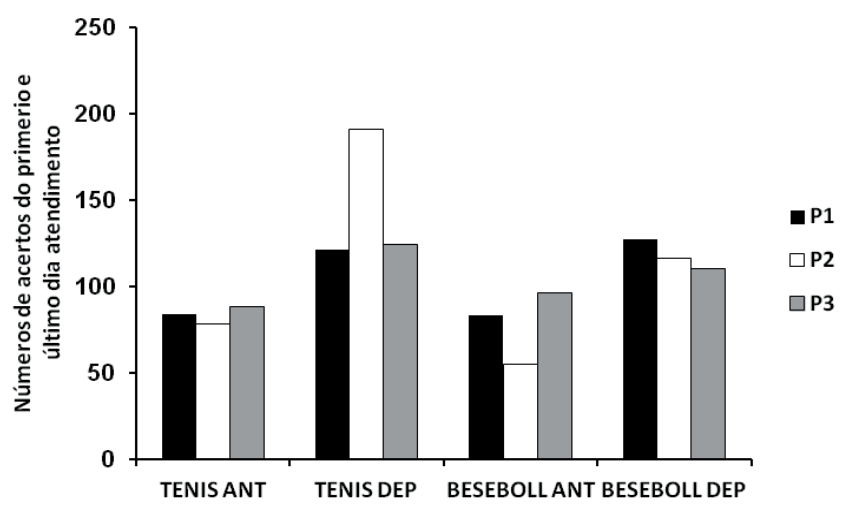

Figura 4. demostra a média e desvio padrão dos escores obtido de cada paciente com relaçáo ao número de acertos do jogo de tênis e beisebol. P1: Paciente 1, P2: paciente 2; P3: paciente 3.

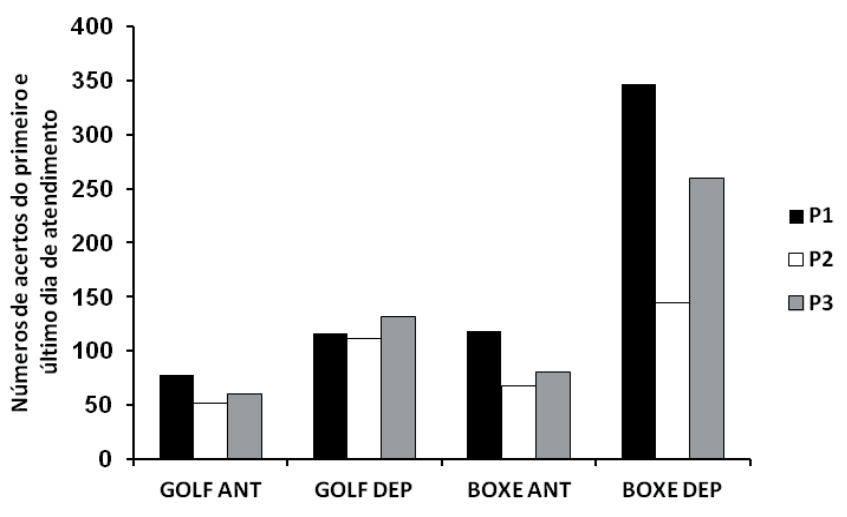

Figura 5. demostra a média e desvio padrão dos escores obtido de cada paciente com relação ao número de acertos do jogo de golf e boxe. P1: Paciente 1, P2: paciente 2; P3: paciente 3. 
cução dos movimentos. Comparando com o presente estudo observa-se que RV promoveu melhora das habilidades funcionais com relação à frequência de uso e a qualidade do movimento destas crianças.

A viabilidade e a eficácia da realidade virtual (RV) em crianças portadoras de paralisia cerebral têm sido exploradas por alguns pesquisadores. Um destes estudos relata que crianças em ambientes virtuais interagem com mais segurança e são capazes de praticar muitas tarefas. Perceberam, que a persistência e o rendimento eram maior quando as crianças tinham algum controle na sua atividade, algo que é proporcionado nos ambientes virtuais, uma vez que o indivíduo pode controlar a execução de suas tarefas por meio de seus movimentos corporais. A RV proporciona um biofeedback visual trazendo estímulos, melhorando a motivação e comportamento para execução das tarefas ${ }^{23,24}$.

Weiss et al. ${ }^{25}$ enfatizaram que os sistemas de captura de movimento tem um grande potencial terapêutico, incluindo melhora da atividade funcional e reabilitação motora. Em relação ao Wii Sports todos os jogos (tênis, boxe, baseball, golf, boliche) proporcionam o controle dos membros superiores, além disso, os jogos em posição ortostática desencadeiam o equilíbrio acarretando a transferência de peso entre as extremidades inferiores. $\mathrm{O}$ jogo de boxe exige a orientação da linha média do tronco e da força dos músculos do tronco, os demais jogos exigem a estabilizaçáo do tronco enquanto se move o membro superior com variados graus de força ${ }^{24}$. Isto foi observado também no presente estudo onde as crianças relataram maior facilidade para realizar movimentos, sentiram que o membro parético estava mais firme para segurar objetos, apresentando maior controle do membro superior na realização das AVD's. Todos os jogos foram realizados na posição ortostática, sendo assim houve também um treino de equilíbrio e descarga de peso nos membros inferiores dos indivíduos que participaram no estudo.

O exagero deste recurso acarreta dores e lesóes musculares com uso contínuo. É importante ter cautela e supervisão na utilização deste dispositivo ${ }^{26}$. Sendo assim, neste estudo, foi realizado o alongamento da musculatura no início e término das sessóes e também orientações adequadas quanto ao manuseio correto deste recurso e a execução de cada atividade, a fim de evitar possíveis dores e lesões musculares.

Griffiths ${ }^{27}$ analisou uma amostra de 22 crianças do gênero feminino com idades entre 9 e 13 anos as quais apresentavam transtornos de déficit de atenção. Sua amostra foi dividida em dois grupos, o primeiro foi submetido a tratamento tradicional e o segundo instruído a jogar jogos de vídeo game. $\mathrm{O}$ grupo submetido à terapia com jogos obteve melhora da capacidade de atenção, impulsividade e hiperatividade, e a uma melhor motivação para realizar a terapia. Os pais relataram que seus filhos apresentaram melhor rendimento na escola após tratamento. Pode-se verificar também, no presente estudo, que ao longo das sessões houve um melhora quanto à atenção contínua, autoconfiança e motivação das crianças. Após o tratamento as mães ressaltaram que elas estavam mais alerta e atentas, tinham mais consciência e movimentavam o membro parético com mais frequência.

You et al..$^{28}$ objetivaram analisar o efeito da terapia virtual na reorganização cortical de uma criança com PC hemiparética. Eles utilizaram como recurso a ressonância magnética funcional antes e após a RV. Concluíram que os ambientes virtuais oferecem estimulação sensório-motora, facilita a ativação de redes cerebrais específicas contribuindo para uma reorganização das áreas corticais. Este fato fornece evidência para o princípio de neuroplasticidade, através de um treinamento constante e repetitivo houve o reaprimoramento cortical. Podemos sugerir no presente estudo que antes da intervenção muitas habilidades funcionais não eram possíveis, depois das sessôes houve melhora das habilidades motoras funcionais, incluindo alcance e manipulação de objetos, alimentação e higienização pessoal.

Jang et al. ${ }^{21}$, analisaram 10 pacientes portadores de AVC, apresentando sequela de hemiparesia, foram submetidos aos efeitos da RV. Analisaram a reorganização cortical e recuperação motora, sendo quantificado e qualificado o uso do membro afetado pela escala do MAL. A intervenção foi realizada por 60 minutos ao dia, cinco vezes por semana durante quatro semanas consecutivas. Após a terapia houve uma reorganização das áreas cerebrais lesionadas, além de uma recuperação funcional do membro acometido. $\mathrm{O}$ uso intensivo do membro afetado 
pode gerar potencialização sináptica eficaz, aumentando assim a prática induzida pela neuroplasticidade. A prática repetitiva e o treinamento são condiçôes prévias para induzir a plasticidade funcional a longo prazo. Neste estudo, pode sugerir que a RV pode ser capaz de plasticidade, pois houve uma repetição da atividade por um período de 10 dias consecutivos, nota-se um ganho das habilidades funcionais do membro parético principalmente na área de autocuidado e melhora funcional durante as AVD's.

Deutsch et al. ${ }^{24}$ analisaram o efeito da RV. Eles utilizaram o sistema de jogos do novo console Wii na reabilitação de um adolescente de 13 anos com diagnóstico de PC diplégica espástica. A intervenção teve como duração 60 a 90 minutos por um período de 10 sessóes. Utilizaram o software de jogos Wii Sports, incluindo o boxe, tênis, baseball e golf. Os jogos foram realizados na posição sentada e ortostática dando auxílio e suporte ao paciente. Este apresentava boa capacidade cognitiva para seguir as instruçóes e realizar os jogos. Concluíram que houve uma melhora no processamento visoespacial, melhora no controle postural, na distribuição de peso e também nas habilidades funcionais do adolescente. Na presente pesquisa utilizou-se o mesmo software de jogos, o boxe, tênis, golf e baseball e após as sessóes todas as três crianças melhoraram suas habilidades funcionais.

Alguns fatores podem ser apontados como limitaçóes do estudo. Destacamos a amostra pequena da população e sua composição formada somente de mulheres, o que pode vir a restringir a generalização dos resultados. Mesmo assim, este estudo traz contribuições importantes, além de abrir possibilidades para novas pesquisas. Todas as três crianças apreciaram a utilização do vídeo-game como ferramenta para o atendimento terapêutico e relataram ao final das sessóes que gostariam de continuar o tratamento. Tais observaçôes demonstram que o sistema de realidade virtual fornece um ambiente motivador e desafiante. Além disso, as mães relataram que durante as duas semanas de tratamento, observaram que as crianças estavam mais atentas, reconheciam e utilizavam mais MS parético durante suas atividades cotidianas. Mostrando que a prática repetitiva de uma tarefa motora pode resultar em substanciais melhoras funcionais.

\section{CONCLUSÃO}

A utilização da Terapia Virtual na reabilitação promoveu ganho funcional, melhora de suas habilidades e destreza para o membro superior de crianças hemiparéticas. Sugere-se a realização de outros estudos com uma amostra maior para confirmar a potencialidade e veracidade dos resultados adquiridos.

\section{REFERÊNCIAS}

1.Costa MCF, Bezerra PP, Oliveira APR. Impacto da hemiparesia na simetria e na transferência de peso: repercussóes no desempenho funcional. Rev Neurocienc 2006;14:10-3.

2.Ortega DS. O Paciente Neurológico/Neurocirúrgico Pediátrico. In: Sarmento GJV. Fisioterapia Respiratória em Pediatria e Neonatologia. Barueri: Manole; 2007 , p. $176-95$.

3.Gomes FL. Fraqueza Muscular. In: Lopes AC, José FF, Lopes Rd. Guias de medicina. Barueri: Manole; 2007, p. 1857-71.

4.Morais GFS, Nascimento LR, Glória AE, Salmela LFT, Paiva CMR, Lopes TAT. A influência do fortalecimento muscular no desempenho motor do membro superior parético de indivíduos acometidos por Acidente Vascular Encefálico. Acta Fisiatr 2008;15:245-8.

5.Nascimento LR, Glória AE, Habib ES. Effects of constraint-induced movement therapy as a rehabilitation strategy for the affected upper limb of children with hemiparesis: systematic review of the literature. Rev Bras Fisioter 2009; 13:97-102.

http://dx.doi.org/10.1590/S1413-35552009005000022

6.Gianni MADC. Paralisia Cerebral - Aspectos clínicos. In: Moura EW, Silva PAC. Fisioterapia: aspectos clínicos e práticos da reabilitação. 3 ed. São Paulo: Artes Médicas; 2005, p.13-25.

7.Marinho APS, Souza MAB, Pimentel AM. Desempenho funcional de crianças com paralisia cerebral diparéticas e hemiparéticas. Rev Cien Med Biol 2008;7:57-66.

8.Carvalho LFA, Affonseca CA, Guerra SD, Ferreira AR, Goulart EMA. Traumatismo Cranioencefálico Grave em Crianças e Adolescentes. Rev Bras Ter Int. 2007;19:98-106.

http://dx.doi.org/10.1590/S0103-507X2007000100013

9. Junior AL. Conduta frente à criança com trauma craniano. J Ped 2002;78:40-7. 10.Jerônimo RA, Lima SMPF. Tecnologias Computacionais e ambientes virtuais no processo terapêutico de reabilitação. Mundo Saúde 2006;30:96-106.

11.Kisner C, Tori R. Introduçâo à Realidade Virtual, Realidade misturada e hiperrealidade. In: Kisner C, Tori R. Realidade virtual: conceitos e tendências. São Paulo: Mania de Livro; 2004, p.3-20.

12.Broeren J. Virtual rehabilitation: Implications for persons with stroke(endereço na internet). Gothenburg, Sweden: Gothenburg University Publications Electronic Archive (GUPEA).(atualizado em 2011; assessado em 2012). Disponível em: http://gupea.ub.gu.se/bitstream/2077/7329/1/PhD_ Thesis_Jurgen_Broeren.pdf.

13.Deuschle VP, Cechella C. O déficit em consciência fonológica e sua relação com a dislexia: diagnóstico e intervenção. Rev CEFAC 2009;11:194-200. http://dx.doi.org/10.1590/S1516-18462008005000001 
14.Silva MFMC, Kleinhans ACS. Processos cognitivos e plasticidade Cerebral na Síndrome de Down. Rev Bras Ed Esp 2006;12:123-38.

15.Vasconselos RLDM, Moura TL, Campos TF, Lindquist ARR, Guerra RO. Avaliação do desempenho funcional de crianças com paralisia cerebral de acordo com os níveis de comprometimento motor. Rev Bras Fisioter 2009;13:390-7. http://dx.doi.org/10.1590/S1413-35552009005000051

16.Fonseca JO, Cordani LK, Oliveira MC. Aplicação do inventário de avaliaçáo pediátrica de incapacidade (PEDI) com crianças portadoras de paralisia cerebral tetraparesia espástica. Rev Ter Ocup USP 2005;16:67-74.

17.Carlberg EB, Vollestad NK. Everyday functioning in Young children with cerebral palsy: functional skills, caregiver assistance, and modifications of the environment. Dev MedChild Neurol 2003;45:603-12.

18. Uswatte G, Taub E. Implications of the Learned Nonuse Formulation for Measuring Rehabilitation Outcomes: Lessons From Constraint-Induced Movement Therapy. Rehabil Psychol 2005;50:34-42.

http://dx.doi.org/10.1037/0090-5550.50.1.34

19. Wolf SL, Lecraw DE, Barton LA, Jann BB. Forced use of hemiplegic upper extremities to reverse the effect of learned nonuse among chronic stroke and head-injured patients. Exp Neurol 1989;104:125-32.

http://dx.doi.org/10.1016/S0014-4886(89)80005-6

20.Paz LPDS. Quantidade e qualidade do uso da extremidade superior parética após acidente cerebrovascular [dissertação]. Campinas: Faculdade de Ciências Médicas da Universidade Estadual de Campinas, Unicamp, 2007.

21.Jang SH, You SH, Hallett M, Cho YW, Park CM, Cho SH. Cortical Reorganization and Associated Functional Motor Recovery After Virtual Reality in Patients With Chronic Stroke: An Experimenter-Blind Preliminary Study. Arc
Phys Med Rehabil 2005;86:2218-23.

http://dx.doi.org/10.1016/j.apmr.2005.04.015

22.Flynn S, Copar S, Ghate N, Harris M, Palma P, Bender A. Effects of Virtual Reality Immersion in Individuals with Central Nervous System Injury (endereço na internet). Philadelphia: Temple University. (atualizado em 2012; acessado em 2012). Disponível em: http://www.temple.edu/ispr/prev_conferences/ proceedings/2007/Flynn,\%20et\%20al.pdf

23. Reid DT. The influence of virtual reality on playfulness in children with cerebral palsy: a pilot study. Occup Ther Inte 2004;11:131-44.

http://dx.doi.org/10.1002/oti.202

24.Deutsch JE, Borbely M, Filler J, Huhn K, Bowlby PG. Use of a Low-Cost, Commercially Available Gaming Console (Wii) for Rehabilitation of an Adolescent With Cerebral Palsy. Phys Ther2008;88:1196-207.

http://dx.doi.org/10.2522/ptj.20080062

25.Weiss PL, Rand D, Katz N, Kizony R. Video capture virtual reality as a flexible and effective rehabilitation tool. J NeuroEngin Rehabil 2004;12:1-12. 26.Ferreira LR, Silva AT, Deamo RA. A utilização da realidade virtual como recurso terapêutico: estudo de caso [dissertação]. Itajubá: Centro Universitario de Itajubá: Universitas, 2008.

27.Griffiths M. The educational benefits of videogames Video games have great positive potential in addition to their entertainment value and there has been considerable success when games are designed to address a specific problem or to teach a certain skill. Edu Health 2002;20:47-51.

28. You SH, Jang SH, Kim YH. Cortical reorganization induced by virtual reality therapy in a child with hemiparetic cerebral palsy. Dev Med Child Neurol 2005; 47:628-35.

http://dx.doi.org/10.1111/j.1469-8749.2005.tb01216.x 